\title{
Antigen Presenting Cell-Mediated Expansion of Human Umbilical Cord Blood Yields Log-Scale Expansion of Natural Killer Cells with Anti-Myeloma Activity
}

\author{
Nina Shah ${ }^{1 *}$, Beatriz Martin-Antonio ${ }^{1}$, Hong Yang ${ }^{1}$, Stephanie $\mathrm{Ku}^{2}$, Dean A. Lee ${ }^{3}$, Laurence J. N. Cooper ${ }^{3}$, \\ William K. Decker ${ }^{2,4}$, Sufang $\mathrm{Li}^{1}$, Simon N. Robinson ${ }^{1}$, Takuya Sekine ${ }^{1}$, Simrit Parmar ${ }^{1}$, John Gribben ${ }^{5}$, \\ Michael Wang ${ }^{6}$, Katy Rezvani ${ }^{1}$, Eric Yvon ${ }^{1}$, Amer Najjar ${ }^{7}$, Jared Burks ${ }^{8}$, Indreshpal Kaur ${ }^{1}$, \\ Richard E. Champlin ${ }^{1}$, Catherine M. Bollard ${ }^{2}$, Elizabeth J. Shpall'
}

1 Department of Stem Cell Transplantation and Cellular Therapy, The University of Texas M.D. Anderson Cancer Center, Houston, Texas, United States of America, 2 Center for Cell and Gene Therapy, Baylor College of Medicine, Houston, Texas, United States of America, $\mathbf{3}$ Department of Pediatrics, The University of Texas M.D. Anderson Cancer Center, Houston, Texas, United States of America, 4 Department of Pathology and Immunology, Baylor College of Medicine, Houston, Texas, United States of America, 5 Institute of Cancer, Queen Mary University of London, Centre for Medical Oncology, Barts and The London School of Medicine, London, United Kingdom, 6 Department of Lymphoma, The University of Texas M.D. Anderson Cancer Center, Houston, Texas, United States of America, 7 Department of Experimental Diagnostic Imaging, The University of Texas M.D. Anderson Cancer Center, Houston, Texas, United States of America, 8 Department of Leukemia Research, The University of Texas M.D. Anderson Cancer Center, Houston, Texas, United States of America

\begin{abstract}
Natural killer (NK) cells are important mediators of anti-tumor immunity and are active against several hematologic malignancies, including multiple myeloma (MM). Umbilical cord blood (CB) is a promising source of allogeneic NK cells but large scale ex vivo expansion is required for generation of clinically relevant CB-derived NK (CB-NK) cell doses. Here we describe a novel strategy for expanding NK cells from cryopreserved CB units using artificial antigen presenting feeder cells (aAPC) in a gas permeable culture system. After 14 days, mean fold expansion of CB-NK cells was 1848-fold from fresh and 2389 -fold from cryopreserved CB with $>95 \%$ purity for NK cells $\left(\mathrm{CD}^{2} 6^{+} / \mathrm{CD}^{-}\right)$and less than $1 \% \mathrm{CD}^{+}$cells. Though surface expression of some cytotoxicity receptors was decreased, aAPC-expanded CB-NK cells exhibited a phenotype similar to CBNK cells expanded with IL-2 alone with respect to various inhibitory receptors, NKG2C and CD94 and maintained strong expression of transcription factors Eomesodermin and T-bet. Furthermore, CB-NK cells formed functional immune synapses with and demonstrated cytotoxicity against various MM targets. Finally, aAPC-expanded CB-NK cells showed significant in vivo activity against $\mathrm{MM}$ in a xenogenic mouse model. Our findings introduce a clinically applicable strategy for the generation of highly functional CB-NK cells which can be used to eradicate MM.
\end{abstract}

Citation: Shah N, Martin-Antonio B, Yang H, Ku S, Lee DA, et al. (2013) Antigen Presenting Cell-Mediated Expansion of Human Umbilical Cord Blood Yields LogScale Expansion of Natural Killer Cells with Anti-Myeloma Activity. PLoS ONE 8(10): e76781. doi:10.1371/journal.pone.0076781

Editor: Evren Alici, Karolinska Institutet, Sweden

Received April 16, 2013; Accepted August 29, 2013; Published October 18, 2013

Copyright: (c) 2013 Shah et al. This is an open-access article distributed under the terms of the Creative Commons Attribution License, which permits unrestricted use, distribution, and reproduction in any medium, provided the original author and source are credited.

Funding: This work was supported by the National Institutes of Health K12 CA088084 (Shah) and Cancer Prevention and Research Institute of Texas RP\#100430 (Shpall). The funders had no role in study design, data collection and analysis, decision to publish, or preparation of the manuscript.

Competing Interests: The authors have declared that no competing interests exist.

*E-mail: nshah@mdanderson.org

\section{Introduction}

Multiple myeloma (MM) is the second most common hematologic malignancy in adults [1]. It is currently considered incurable, even after high dose chemotherapy and autologous hematopoietic stem cell transplantation (HSCT) [2]. Natural killer (NK) cells are $\mathrm{CD}_{56}{ }^{+} / \mathrm{CD} 3^{-}$cytotoxic lymphocytes that are increasingly recognized as a potent cellular therapy. NK cells have been shown to be active against $\mathrm{MM}$ in several preclinical studies [3,4]. In addition, a relative decrease in $\mathrm{NK}$ cell frequency or function in MM patients has been shown to correlate with more advanced disease or poorer outcome $[5,6]$.

NK cell cytotoxic activity can be triggered by cytokines, antibodies or a shift in the balance between their activating and inhibitory receptors. Specifically, NK cells are cytotoxic to cells lacking appropriate self-major histocompatibility complex (MHC) class I molecules via disinhibition of the killer immunoglobulin-like receptor (KIR). This forms the basis for the "missing self" hypothesis [7] and is thought to mediate donor $\mathrm{NK}$ cell alloreactivity in the setting of allogeneic HSCT. However the precise role of KIR-ligand mismatch in HSCT is not known. In some patients treated with allogeneic-HSCT, PB-NK cell alloreactivity as determined by missing KIR ligands appears to predict reduced rates of relapse and graft versus host disease (GVHD) [8,9]. Additionally, in MM patients undergoing matched allogeneic-HSCT, an activated donor KIR haplotype $(\mathrm{Bx})$ has been associated with a significantly lower risk of relapse and better PFS [10]. In contrast, other studies have suggested that the effect of KIR-ligand incompatibility is not consistent, particularly as it relates to conditioning regimen, donor source and GVHD outcomes [11,12,13,14].

Although allogeneic NK cells appear promising in MM, autologous PB-NK cells from MM patients appear to be hypofunctional [15]. This may be due to inhibitory cytokines 
such as TGF- $\beta$, IL-6 and IL-10 present in the MM microenvironment $[16,17,18]$ or dysregulation of IL-15 signaling in favor of MM cells over activation of NK cells $[19,20]$. While some preclinical studies suggest that this NK cell dysfunction can be reversed via ex vivo expansion/activation $[4,21,22]$, the potentially unpredictable nature of autologous NK cells from heavily pretreated patients warrants further optimization of techniques for allogeneic adoptive NK cell therapy. Furthermore, in advanced disease states, MM cells may upregulate Class I expression [23]. This suggests that KIR-MHC class I mismatched, allogeneic NK cell therapy would be advantageous over autologous NK cell therapy, as allogeneic NK cells would be less inhibited by cognate MHC class I in contrast to autologous NK cells.

To date, the majority of clinical trials of NK cell therapy for various malignancies have used allogeneic $\mathrm{PB}$ as a source of $\mathrm{NK}$ cells. We are interested in NK cells derived from human umbilical cord blood $(\mathrm{CB})$ as an alternative and more readily available source of NK cells. Our group has previously demonstrated that $e x$ vivo expansion with IL-2 activates otherwise quiescent CB-NK cells. These GB-NK cells exhibit a mature phenotype, similar to PB-NK cells, and are as active as PB-NK cells against leukemia targets [24].

The limited number of NK cells in an unmanipulated CB unit requires an efficient and robust NK cell ex vivo expansion strategy. Several groups have recently reported expansion of PB-NK cells using genetically engineered artificial antigen presenting cells (aAPCs) derived from the K562 cell line [25,26]. In this study, we build upon recently developed technology with aAPCs [26] and describe a novel technique for expanding CB-NK cells for use in MM. This good manufacturing practice (GMP)-compliant method yields clinical scale expansion of phenotypically mature GB-NK cells which are cytotoxic to MM cells in vitro and demonstrate in vivo anti-MM activity in a xenogenic model. Taken together, our results provide the basis for further exploration of CB-NK cell therapy for patients with MM.

\section{Materials and Methods}

\section{Ethics Statement}

All research involving human materials was approved by the MD Anderson (MDACC) Institutional Review Board (IRB). Cord blood units were obtained from healthy donors who gave written informed consent. All animal work was performed under an MDACG Institutional Animal Care and Use Committee (IAGUC)-approved protocol specific to this study.

\section{Cells and Cell Lines}

K562-based aAPCs expressing membrane bound IL-21 "Clone 9.mbIL21" were generously provided by Dr. Laurence Cooper (MDACG, Houston TX). Clone 9.mbIL21 cells express membrane-bound IL-21, 41BB ligand, CD64 (Fc $\gamma$ RI) and CD86. This cell line has recently been shown to promote $\mathrm{PB}$ NK cell expansion [26]. and is GMP-grade for clinical use. Targets for NK cell functional assays consisted of K562 cells (American Type Culture Collection (ATCG), Rockville, MD) and MM cell lines RPMI 8226 (ATCG), ARP-1 (Multiple Myeloma Research Center, Little Rock AK), and U266 (ATCG). Autologous, unselected $\mathrm{CB}$ cells (from the same $\mathrm{CB}$ unit as the NK cells) were used as a negative control for ${ }^{51}$ chromium $(\mathrm{Cr})$ experiments.

\section{Generation of eGFP-FFLuc-expressing ARP-1 Cell Line for in vivo Experiments}

The generation of retrovirus vectors encoding green fluorescent protein (eGFP)-Firefly Luciferase (eGFP-FFLuc) and production of transient retroviral supernatant have been previously described $[27,28]$. Briefly, the fusion protein eGFP-FFLuc was cloned into an SFG retroviral vector and retroviral supernatant was produced using 293-T cells co-transfected with the following retroviral vectors: eGFP-FFLuc SFG plasmid, the Peg-Pam-e plasmid containing the sequence for the MoMLV gag-pol and the RDF plasmid encoding for the RD1 14 envelope. Retroviral supernatant was collected at 48 and 72 hours after transfection and stored at $-80^{\circ} \mathrm{C}$ for further use. For the generation of eGFP-FFLucexpressing ARP-1 tumor cells, 50,000 cells were plated in presence of retroviral supernatant encoding eGFP-FFLuc in one well of a 24-well plate pre-coated with recombinant fibronectin fragment (CH-296; Takara Shuzo, Otsu, Japan). Transduced ARP-1 cells were expanded and eGFP expression evaluated by fluorescenceactivated cell sorter (FACSCalibur; Becton-Dickinson (BD), San Jose, CA) analysis, whereas expression of FFLuc was detected using D-luciferin (Promega, Madison, WI) and bioluminescence measured with a luminometer (Modulus; Turner BioSystems, Sunnyvale, CA). Because of the absence of selection gene in the eGFP-FFLuc retroviral construct, single cell cloning of the ARP-1transduced cells was performed to isolate and expand an ARP-1 clone (clone \# 24) with high level of eGFP and FFLuc expression. As ARP-1 expresses both CD138 and kappa light chain [29,30], Clone 24 was further validated by FACS analysis for CD138 and Kappa light chain expression and ELISA for Kappa light chain secretion.

\section{Isolation and Expansion of Umbilical Cord Blood-derived NK Cells}

$\mathrm{CB}$ units were obtained from healthy donors who gave informed consent under MDACG IRB-approved protocols. Culture media was comprised of $45 \%$ RPMI-1640 (Cellgro, Manassas, VA) and $45 \%$ Click's media (Irvine Scientific, Santa Ana, CA) supplemented with 10\% AB human serum (Atlanta Biologicals, Lawrenceville, GA) and 100 IU/mL IL-2 (Proleukin; Chiron, Emeryville, CA).

$\mathrm{CB}$ mononuclear cells (MNCs) were isolated from fresh or frozen $\mathrm{CB}$ units by ficoll density gradient centrifugation. Twenty million MNCs were plated in $400 \mathrm{~mL}$ media in a GP500 gas permeable bioreactor (Wilson Wolf Corporation, New Brighton, MN) with irradiated (100 Gy) aAPG feeder cells (2:1 feeder cell:MNC ratio) at $37^{\circ} \mathrm{C}$. IL-2 was replenished every $2-3$ days. On day 7, cultured cells were CD3-depleted via immunomagnetic depletion according to manufacturer's instructions (Miltenyi Biotech, Auburn, CA). Remaining cells were then re-plated in the same conditions, re-stimulated with aAPC feeder cells and cultured for an additional 7 days (Figure 1). Flow cytometric analysis was performed on Days 0, 7 and 14 during the expansion. NK cell number was determined by multiplying the live total nucleated cell count by the percentage of $\mathrm{CD} 56^{+} / \mathrm{CD} 3^{-}$cells. Differences in cell growth were calculated using a 2-tailed student's t-test (Microsoft Excel 2010, Redmond, WA).

\section{Original Expansion Techniques}

For comparison, CB-NK cells were also expanded by a method already known to be successful in our laboratory [24]. Fresh CB MNCs were isolated as above and then subjected to CD56 ${ }^{+}$ immunomagnetic selection. These cells were then suspended at $1 \times 10^{6}$ cells $/ \mathrm{mL}$ culture media with IL-2 at $500 \mathrm{IU} / \mathrm{mL}$. The cells were cultured for 14 days at $37^{\circ} \mathrm{C}$; IL-2 was replenished every 2-3 days. 


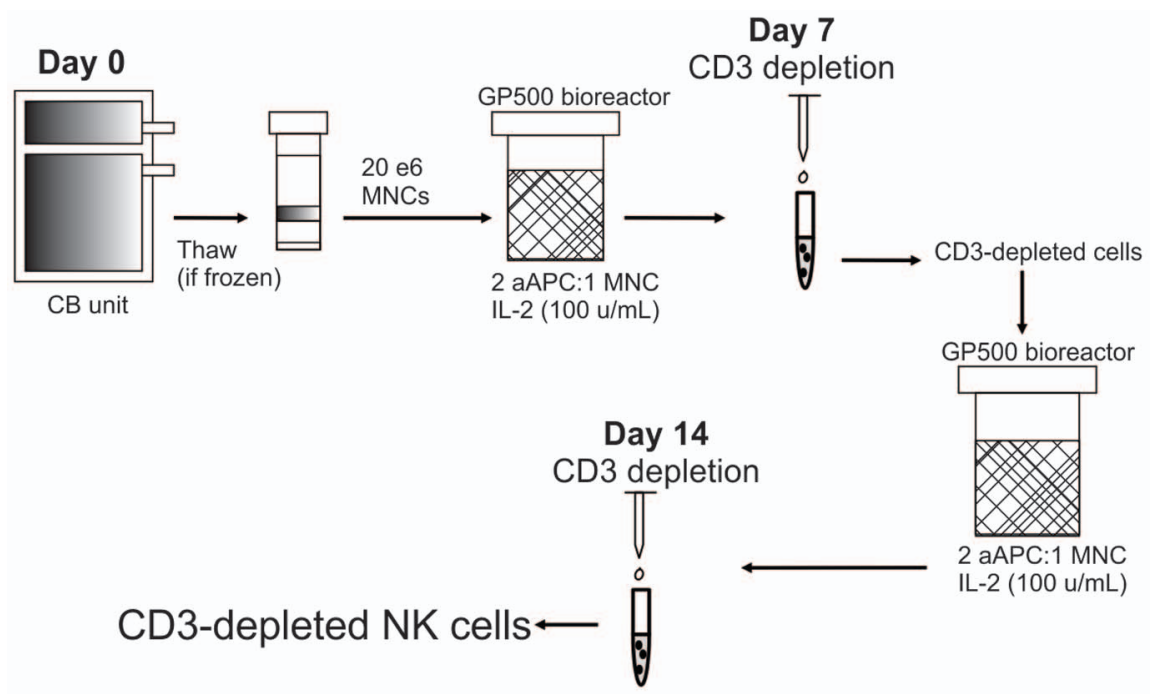

Figure 1. Culture of CB-NK cells. Unselected CB MNCs were cultured for 7 days in a GP500 bioreactor with IL-2 (100 IU/mL) and aAPCs at 2:1 aAPC:MNC ratio. Cells were immunomagnetically CD3-depleted on Day 7 and re-cultured in same conditions for an additional 7 days. On day 7 cells were again CD3-depleted and subject to phenotypic and functional studies. doi:10.1371/journal.pone.0076781.g001

\section{NK Cell Phenotyping via Flow Cytometry}

The following antibodies were used: FITC-conjugated CD45, CD158a, CD158b, CD94; PE-conjugated CD16, CD56, NKp30, NKp46, NKp44, NKG2C; PerCP-conjugated CD3; APC-conjugated CD56, NKG2A; Alexa Fluor 647- conjugated Eomesodermin, T-bet (BD Biosciences); FITC-conjugated CD158el (BioLegend, San Diego, CA); aAPC-conjugated NKG2A (Beckman Coulter, Brea, CA). Intracellular staining for Eomes and T-bet was performed per manufacturer's guidelines (BD Cytofix/Cytoperm, BD Biosciences). Data were acquired by the BD FACSCalibur device using BD CellQuest-Pro software. Flow cytometry analysis was performed using CellQuest and FlowJo (Tree Star, Ashland, OR) software. Differences in MFI were calculated using a two-sided paired t-test (Microsoft Excel 2010).

\section{Immunofluorescence and Confocal Microscopy Image Acquisition}

Immunofluorescent labeling was performed as previously described [31]. Target cells were labeled with CellTracker Blue CMAC (7-amino-4-chloromethylcoumarin, Molecular Probes, Eugene, OR). NK cell-target cell conjugates were formed by suspending equal volumes and cell numbers of NK effector cells and target cells $\left(5 \times 10^{6} / \mathrm{mL}\right)$ in culture media for $15 \mathrm{~min}$ at $37^{\circ} \mathrm{C}$. Cells were then transferred onto microscope slides using a cell concentrator (Cytofuge 2, IRIS International, and Chatsworth, CA), fixed with $3 \%$ methanol-free formaldehyde and then permeabilized. NK effector cell F-actin was stained with rhodamine-phalloidin (Molecular Probes, Invitrogen, Carlsbad, CA). Images were acquired using an Olympus IX81 microscope (Center Valley, PA).

\section{NK Cell ${ }^{51} \mathrm{Cr}$ Cytotoxicity Assay}

Serial dilutions of NK cells were co-incubated in triplicate for 4 hours with $5000{ }^{51}$ Cr-labeled target cells (Amersham Pharmacia Biotech, Piscataway, NJ), in a total volume of $100 \mu \mathrm{l}$ in a Vbottom 96-well plate (Corning, Corning, NY). Thereafter, supernatants $(50 \mu \mathrm{l})$ were harvested and transferred to a LumaPlate-96 (Perkin-Elmer, Waltham, MA). After drying overnight, ${ }^{51} \mathrm{Cr}$ release was measured on a TOPCount NXT microplate scintillation and luminescence counter (Perkin-Elmer). Cytotoxicity was determined by the formula: cytotoxicity $=($ sample valuespontaneous lysis $) /($ max-lysis-spontaneous lysis $) \times 100 \%$.

\section{ARP-1 Myeloma Murine Model}

NOD/SCID IL-2R $\gamma^{\text {null }}$ (NSG) mice (Jackson Laboratories, Bar Harbor, ME) were irradiated with $300 \mathrm{cGy}$ and inoculated with $1 \times 0^{6}$ eGFP-FFLuc -transduced ARP-1 cells (Clone 24) intravenously on day -1 . Where indicated, $10 \times 10^{6}$ ex vivo, fresh, aAPCexpanded $\mathrm{CB}$ NK cells were given retro-orbitally on days 0,12 and 19 with IL-2 (2000 IU intrapertioneally (IP) three times per week). Mice were subjected to twice weekly bioluminescence imaging (BLI) and weekly serum kappa light chain measurements. Prior to image acquisition mice were anesthetized with $2 \%$ isoflurane in $98 \%$ oxygen. BLI was performed using a Xenogen IVIS 200 system (Caliper, Waltham, MA) 10 minutes following a $100 \mu \mathrm{L}$ IP injection of D-luciferin $(20 \mathrm{mg} / \mathrm{mL}$ PBS $)$. BLI images were acquired at 5-minute exposures and superimposed on bright field photographs of the animals. Signal quantitation in photons/ second $(\mathrm{p} / \mathrm{s})$ was performed by determining the photon flux rate within standardized regions of interest (ROI) using Living Image software (Caliper). Serum kappa levels were measured by a commercially available enzyme-linked immunosorbent assay (ELISA) kit (Bethyl Laboratories, Montgomery, TX) according to manufacturer's instructions. Results reported are a representative experiment with 5 mice in each group. Differences in BLI and serum kappa levels were calculated using a 2-tailed student's t-test (Microsoft Excel 2010). Survival was calculated using the KaplanMeier method (SAS statistical software, version 9.2, Cary, NG).

\section{Results}

aAPC-mediated CB-NK Expansion from Fresh or Cryopreserved CB Units yields Significantly Greater Fold Expansion of NK Cells than Expansion of $\mathrm{CD} 56^{+}$Cells with IL-2 Alone

In comparison with our original expansion approach of CD56selected cells cultured with IL2 alone, culture of either fresh or frozen CB MNCs with aAPC feeder cells resulted in greater 
expansion of NK cells after culture for 14 days $(\mathrm{p}<0.05$ for both fresh or frozen conditions, Figures $2 \mathrm{~A}$ and $2 \mathrm{~B}$ ). Culturing of fresh CB MNGs $(n=8)$ with aAPC feeder cells yielded a mean fold expansion of 1848 fold (609 fold -4778 fold) while culturing of frozen CB MNGs $(n=6)$ with feeder cells yielded a mean fold expansion of 2389 fold (103 fold -4931 fold). This was in comparison to 20 fold ( 11 fold -27 fold) expansion from culture of fresh GD56 ${ }^{+}$-selected cells with IL-2 alone $(n=3)$. The difference in NK cell yield was apparent by day 7 for the fresh CB culture with aAPC feeders $(\mathrm{p}<0.05)$ but did not reach statistical significance for the frozen $\mathrm{CB}$ condition until day $14(\mathrm{p}=0.06$ at day 7). As seen in Figure 2C, the final culture contained very few $(\leq 1 \%) \mathrm{CD}^{+}$cells and this was not significantly different between the 3 culture conditions: mean value of $0.44 \% \mathrm{CD} 3^{+}$cells from the culture with IL-2 alone, $0.74 \% \mathrm{CD}^{+}$cells from fresh $\mathrm{CB}$ MNCs with aAPC feeders and $0.66 \% \mathrm{CD}^{+}$cells from frozen $\mathrm{CB}$ MNCs with aAPC feeders ( $\mathrm{p}>0.5$ for all comparisons).

\section{aAPC-mediated Expansion Yields a Pure Population of NK Cells with a Mature Phenotype}

As seen in Figure 3A, co-culture of CB MNCs with IL-2 and aAPC feeder cells yielded a population that was pure for NK cells at the end of the 2 week expansion period. After CD3-depletion, $96 \%$ of cells were $\mathrm{CD}^{+} 6^{+} / \mathrm{CD}^{-}$and less than $1 \%$ were $\mathrm{CD} 3^{+}$. CB-NK cells expanded with aAPGs demonstrated a CD56 ${ }^{\text {hi }}$ phenotype similar to CB-NK cells expanded with IL-2 alone. Of note, culture of unselected CB MNCs with IL-2 and soluble IL-21 yielded a relatively pure $\mathrm{CD} 56^{+} / \mathrm{CD} 3^{-} \mathrm{NK}$ cell population but with limited expansion of cells (mean expansion of 14 fold, data not shown). In addition, after log-fold expansion, aAPC-expanded CB-NK cells did not appear exhausted; rather, CB-NK cells continued to strongly express Eomesodermin and T-bet, transcription factors recently recognized as necessary for NK cell maturation and activation [32,33] (Figure 3B). Interestingly, the surface expression of NK cytotoxicity receptors (NCRs) NKp30, NKp46 and NKp44 was significantly lower for aAPC-expanded CB-NK cells versus IL-2-expanded CB-NK cells ( $p \leq 0.05$ for all three NCRs). However, the expression of KIR antigens, NKG2A, co-receptor CD94 and the activating receptor NKG2C was similar between the two expansion methods (Figure 3G).

\section{CB-NK Cells Cultured with aAPCs Demonstrate in vitro Anti-myeloma Activity}

In order to kill targets, NK cells must directly contact the cell of interest and form the "NK immune synapse" (NKIS) [34,35]. Our lab has previously demonstrated that expansion of CB-NK cells is necessary to repair the defective NKIS exhibited by naive CB-NK cells [24]. To demonstrate that this synapse ability is maintained in CB-NK cells expanded with aAPG feeder cells, we performed a series of synapse assays with various MM targets. As shown in Figure 4A, NK cells cultured with aAPC feeder cells formed a functional NKIS (demonstrated by F-actin polarization) with the classic NK cell target K562, MM cell lines RPMI 8226, aARP-1 and U266.

To demonstrate the functionality of CB-NK cells expanded with aAPC feeder stimulation, we performed a standard ${ }^{51} \mathrm{Cr}$ cytotoxicity assay. aAPC-expanded CB-NK cells were cytotoxic to all of the MM cell line targets (Figure 4B). Furthermore, despite the differences in phenotype with regard to the NCRs, in comparison with CB-NK cells expanded with IL-2 alone, the aAPC-mediated expanded CB-NK cells demonstrated equal or greater cytotoxicity against K562 (Figure 4C). This finding was
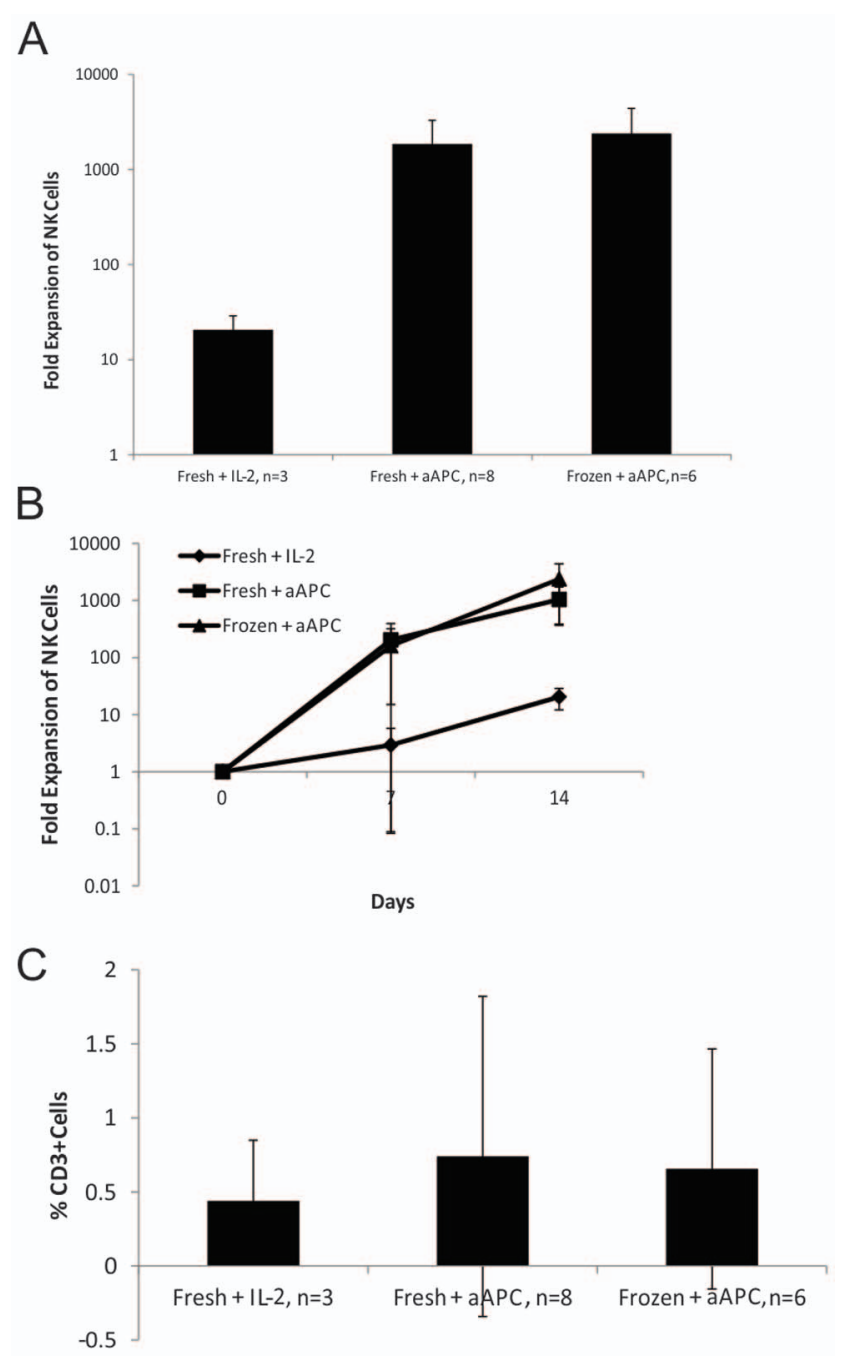

Figure 2. Co-culture of CB MNCs with IL-2 and aAPCs yields significantly greater expansion of NK cells than culture with IL2 alone. A. Mean fold growth of $\mathrm{CD}^{2} 6^{+} / \mathrm{CD}^{-}$NK cells from 8 fresh and 6 frozen cord blood expansions with aAPCs and IL-2 versus 3 expansions with IL-2 alone (14 day culture). B. Time course of NK cell growth over 14 day culture between all 3 conditions. By day 7 , the fresh CB aAPC-containing culture demonstrated greater NK cell growth than culture with IL-2 alone $(p<0.05)$. The frozen CB showed a similar trend at day 7 , which did not reach statistical significance $(p=0.06)$. C. All three culture conditions yielded comparable, low percentages of $\mathrm{CD}^{+}$ cells: $0.44 \%, 0.74 \%$ and $0.66 \% \mathrm{CD}^{+}$cells from the culture with IL-2 alone, fresh CB MNCs with aAPC feeders or frozen CB MNCs with aAPC feeders respectively ( $p>0.5$ for all comparisons). Mean $+/-$ SD is shown for each figure. $\mathrm{P}<0.05$ where indicated $(*)$. doi:10.1371/journal.pone.0076781.g002

consistent across the MM cell lines as well (Figure S1). Neither of the CB-NK preparations demonstrated autologous cytotoxicity.

\section{Treatment with Expanded CB-NK Cells Delays Development of Myeloma in a Murine Model}

To investigate whether ex vivo expanded CB-NK cells can inhibit the growth of MM cells in vivo, we studied NSG mice treated with GFP firefly luciferase-transduced ARP-1 cells (Clone 24). Using the bioluminescent signal intensity as a surrogate for tumor cell density, serial images demonstrated that mice treated with CB-NK cells had a delay in the onset of MM (Figure 5A). After 1 week, the signal intensity $(\mathrm{p} / \mathrm{s})$ was significantly greater in those mice who 
A

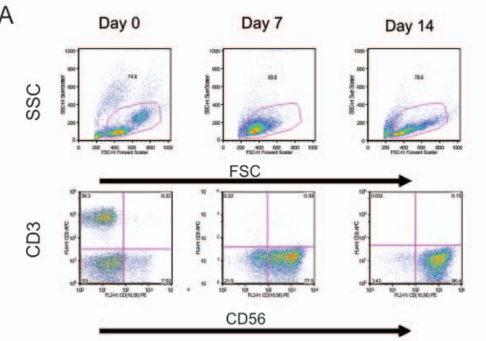

B

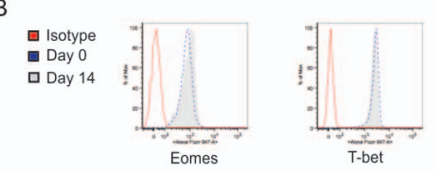

C

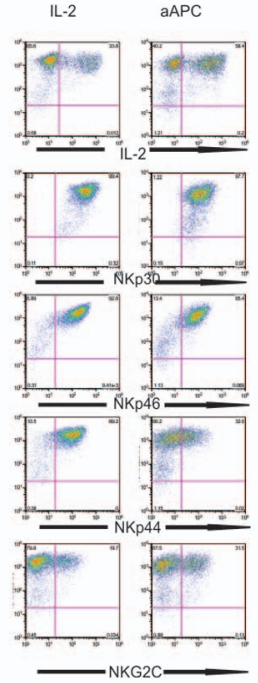

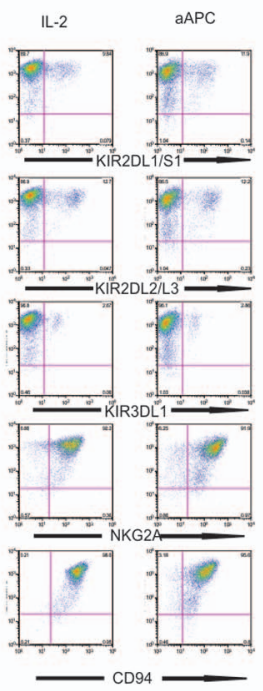

D

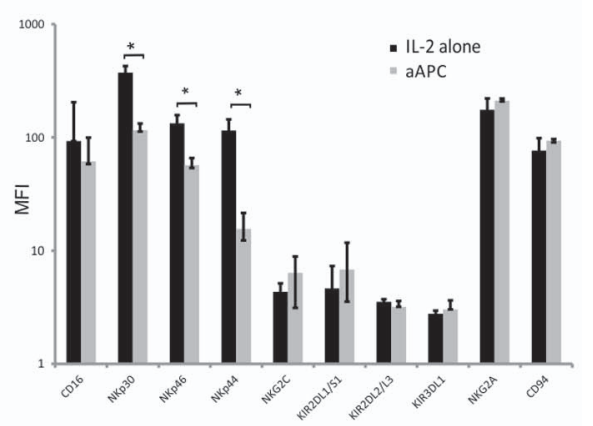

Figure 3. Phenotype of CB-NK cells cultured with aAPCs. A. Over the 14-day expansion, CB-NK cells cultured with aAPC feeder cells demonstrated a progressively pure, $\mathrm{CD}_{56}{ }^{+} / \mathrm{CD} 3^{-}$population, (representative dot plots of 17 expansions). B. aAPC-expanded CB-NK cells maintained Eomesodermin ${ }^{\text {hi }}$ and T-bet ${ }^{\text {hi }}$ phenotype after expansion. Representative histograms from 3 different CB-NK expansions; cells are gated on the live CD56 ${ }^{+}$population. C. CB MNCs from the same CB unit were expanded with aAPCs +IL-2 or IL-2 alone ( $\mathrm{n}=3$ separate CB units). Representative dot plots of NK cell surface receptor expression on day 14 are shown. D. By median fluorescence intensity (MFI), aAPC-expanded CB-NK demonstrated a decreased surface expression of the NCRs NKp30, NKp46 and NKp44. However there was a similar expression between the conditions of the KIR antigens, inhibitory receptor NKG2A, co-receptor CD94 and activating receptor NKG2C) ( $n=3$ paired expansions, mean $+/-S D$ is shown, $p \leq 0.05$ where indicated).

doi:10.1371/journal.pone.0076781.g003

received Clone 24 ARP-1 cells alone versus those who received Clone 24 ARP-1 cells and CB-NK cells (Figure 5B, p $<0.05$ from Day 8-22) This was consistent with the ELISA analysis of serum kappa light chains; mice receiving Clone 24 ARP-1 cells alone had significantly more measurable serum kappa than mice who received Clone 24 ARP-1 cells and CB-NK cells, (Figure 5C, $\mathrm{p}<0.01$ at each time point). Finally, there was also a difference in survival between the 2 groups with a median survival of 31 days in the mice who received Clone 24 ARP-1 cells alone versus 38 days for the mice who received Clone 24 ARP-1 cells and CB-NK cells, (Figure 5D, $\mathrm{p}=0.003$ ).

\section{Discussion}

To our knowledge, this is the first study exploring ex vivo expanded CB-NK cells for the treatment of MM. Clinical trials with allogeneic HSCT for MM consistently show an enhanced complete remission rate in comparison with autologous HSCT regimens $[36,37,38]$, suggesting a true graft versus $\mathrm{MM}$ effect. However, this benefit is off-set by increased treatment-related mortality associated with GVHD [39]. MM is thus an ideal disease candidate for NK cell therapy: in comparison with a $\mathrm{T}$ cell replete allograft, NK cells exert an allogeneic graft versus tumor effect but do not appear to increase the risk of GVHD [40,41]. Indeed a clinical trial with allogeneic PB-derived NK cells for MM has demonstrated safety and no increase in GVHD [42], though the role of KIR-HLA I incompatibility on NK cell alloreactivity remains to be defined.

The in vitro and in vivo data presented here support the use of CB-NK cells against MM. Expanded CB-NK cells exhibited impressive cytotoxicity and immune synapse formation against MM targets. In addition, CB-NK cells were able to significantly delay establishment of disease in a murine MM model. The eventual tumor burden in our in vivo model suggests that cellular therapy would likely have greatest success if administered in combination with other conventional therapies, which could include alkylating or immunomodulatory agents. In addition, the timing of serial NK cell doses may be further optimized to exert greater anti-tumor activity, as has been done in a similar in vivo assay [4].

In comparison to expansion with IL-2 alone, CB-NK cells expanded with aAPCs demonstrated a decreased surface expression of the activating NCRs NKp30, NKp46 and NKp44. However the expression of KIR antigens, inhibitory receptor NKG2a, co-receptor CD94 and activating receptor NKG2C was similar between the 2 conditions. The reason for the decrease in NCR expression is not completely clear. It is possible that the interaction between the CB-NK cells and the K562-based aAPCs during co-culture mediated a transfer of the receptors to the target cells, as has been seen with other NK cell receptors and target cell lines [43]. Interestingly, the differences in NCR surface expression did not appear to impair the functional cytotoxicity of the aAPCexpanded CB NK cells, suggesting that the gain in cell number is not accompanied by a compromise in function. In addition, aAPC-expanded CB-NK cells showed preservation of Eomesodermin and T-bet expression, two transcription factors which have recently been recognized as integral to NK cell function $[32,44,45]$. Recent murine studies have reported that downregulation of these two transcription factors in NK cells following adoptive NK cell transfer and homeostatic proliferation is accompanied by an exhausted phenotype and limited NK cell anti-tumor activity [32]. While one might expect a similar reduction of Eomesodermin and T-bet expression after the logfold expansion of our CB-NK, this was not the case. Additional in vivo studies are in progress to investigate if expanded $\mathrm{CB}$ NK cells are intrinsically less susceptible to exhaustion and more likely to maintain the expression of these transcription factors following adoptive transfer.

The challenge of expanding allogeneic NK cells to a clinically relevant dose remains, as does finding the appropriate donor, if 
A

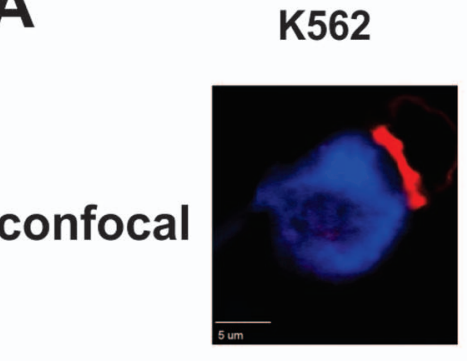

RPMI 8226
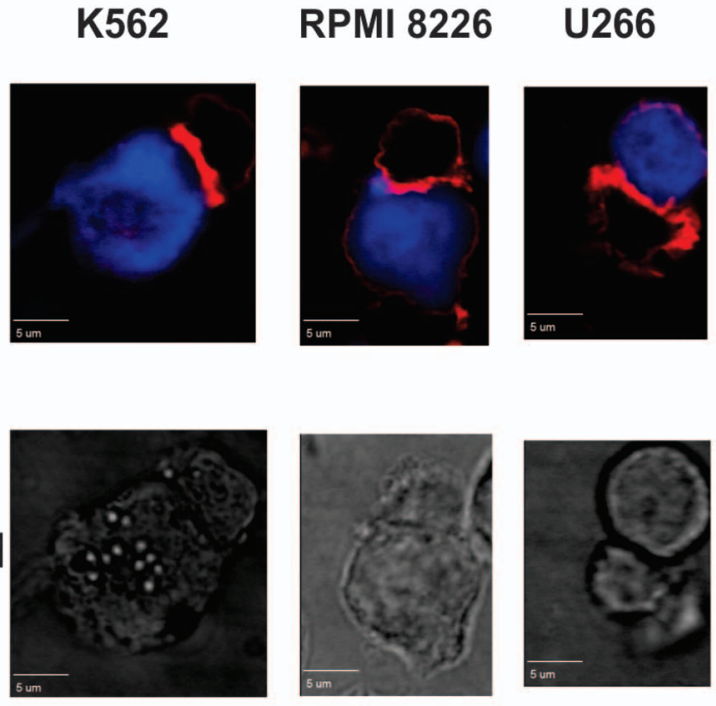

brightfield

B

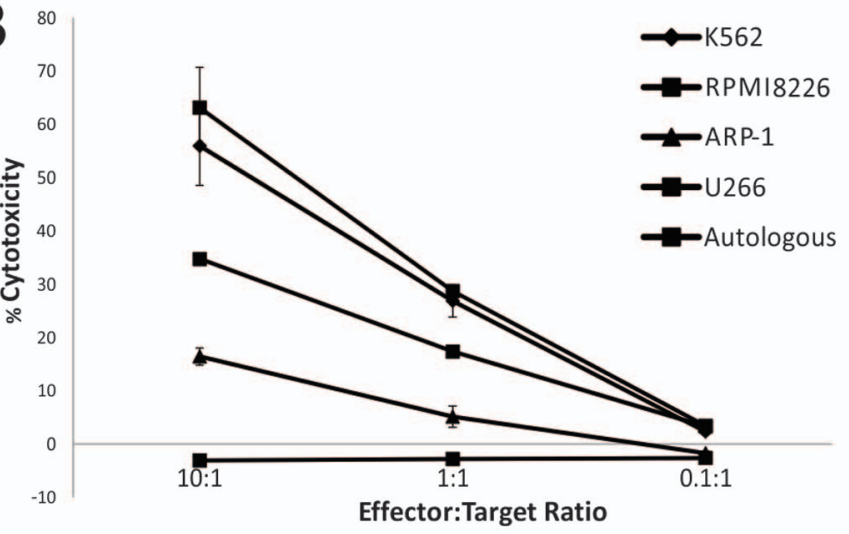

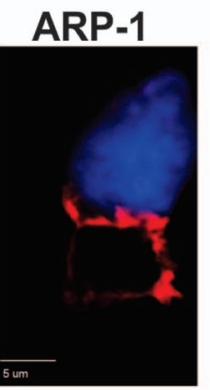

No synapse
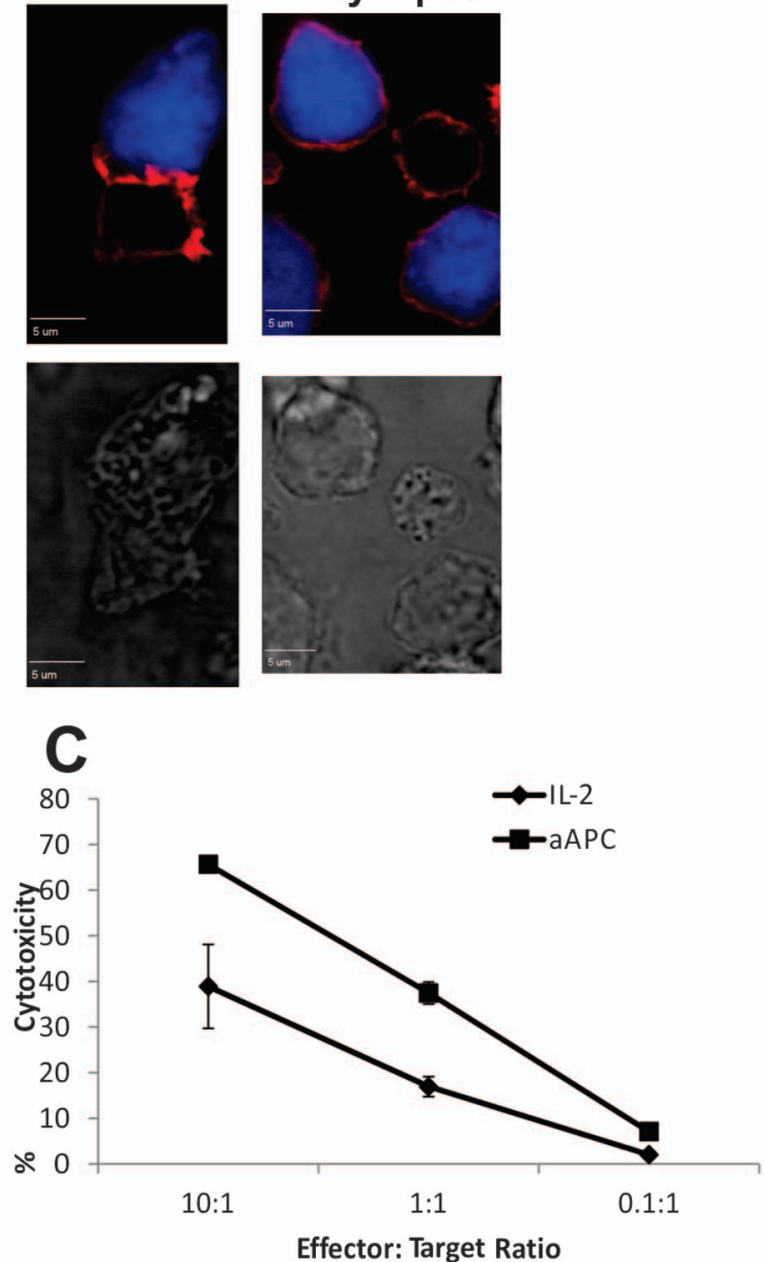

Figure 4. aAPC-expanded CB-NK cells form immunological synapses with and are cytotoxic against myeloma targets. A. CMAClabeled tumor targets (blue) were incubated at a 1:1 ratio with aAPC-expanded CB-NK cells for 15 minutes. Conjugates were then fixed, permeabilized and stained for NK effector cell F-actin with rhodamine-phalloidin (red). Confocal and brightfield images were acquired; representative images from each slide are shown. AAPC-expanded CB-NK cells form immune synapses with the classic NK target K562 as well as a variety of MM cell lines. B. aAPCexpanded CB-NK cells were co-incubated in triplicate for 4 hours with ${ }^{51} \mathrm{Cr}$-labeled target cells at ratios as shown. Supernatants were then harvested and analyzed the next day for ${ }^{51} \mathrm{Cr}$ content. \% Cytotoxicity $=$ (sample value-spontaneous lysis)/(max-lysis-spontaneous lysis) $\mathrm{x} 100 \%$. CB-NK cells demonstrate dose-dependent cytotoxicity against K562 (classic NK cell target) and MM cells lines RPMI 8266, ARP-1 and U266 (representative of n>3 assays for each cell line). C. aAPC-Expanded CB-NK cells displayed equal or more cytotoxicity against K562 cells versus CB-NK cells expanded with IL-2 alone (representative from $n=4$ assays).

doi:10.1371/journal.pone.0076781.g004

indeed the activity of these cells depends on mismatch between donor KIR and recipient HLA I. Here we demonstrate that CB can serve as a reliable source of $\mathrm{NK}$ cells for adoptive cellular immunotherapy. In translating our findings to the clinic, it should be noted that, from $20 \times 10^{6}$ MNCs (approximately $10 \%$ of a clinical CB unit), our culture system would allow for the generation of approximately $1.4 \times 10^{9} \mathrm{NK}$ cells for infusion, or $1.9 \times 10^{7} \mathrm{NK}$ cells $/ \mathrm{kg}$ for an average $70 \mathrm{~kg}$ adult. This is over 18 fold higher than the growth seen with $\mathrm{CD}_{5} 6^{+}$selected cells expanded with IL-2 alone. Additionally, this NK product is relatively pure, with only $6 \times 10^{4} \mathrm{CD} 3^{+}$cells $/ \mathrm{kg}$, thus reducing the potential for GVHD. In comparison with other cryopreserved CBNK culture systems $[46,47,48]$, the method described in this paper has several advantages. First, it requires only two weeks of culture, which could minimize both the cost and potential for microbial contamination seen with longer duration cultures. In addition, this system requires only a fraction of the $\mathrm{CB}$ unit. A minimum of $2 \times 10^{8} \mathrm{CB}$ MNCs are typically obtained from a frozen $\mathrm{CB}$ unit; thus the NK dose could potentially be increased by at least 10 -fold, or a total of $1.9 \times 10^{8} \mathrm{NK}$ cells $/ \mathrm{kg}$. As CB units can be thawed in fractions, this would allow for consideration of serial doses of $\mathrm{NK}$ cell therapy to enhance anti-tumor efficacy.

CB-NK cells could be considered a reasonable alternative to PB-NK cells for adoptive transfer. The potential benefits of expanded NK cells from $\mathrm{CB}$ over $\mathrm{PB}$ include the lower rates of acute GVHD seen in the allogeneic HSCT setting [49,50,51] as well as rapid availability, with over 600,000 banked units worldwide [52]. In addition, CB-NK cells do not require collection from a live donor. Finally, for those patients who do not have a readily available family donor, the $\mathrm{CB}$ pool provides a unique opportunity to find a suitably matched allograft. 
A

Day 4

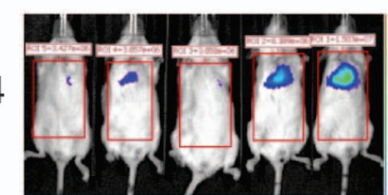

Day 8

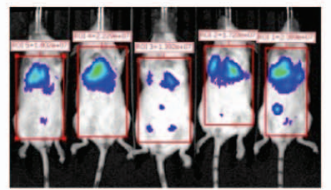

Day11

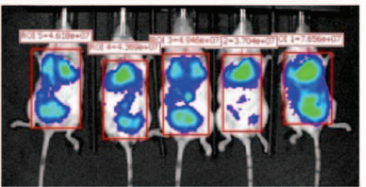

Day 15

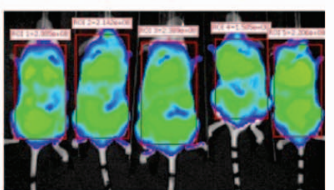

Day 18

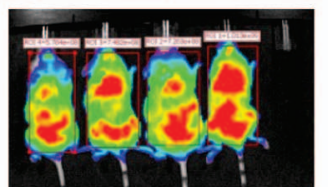

Day 22

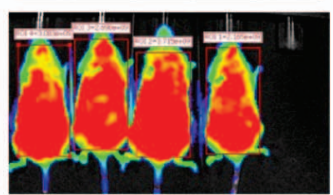

ARP-1
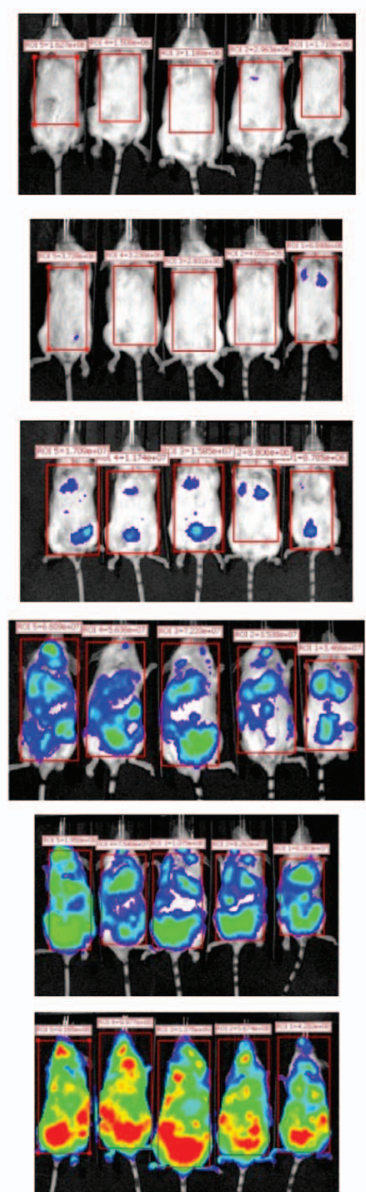

ARP-1 +NK
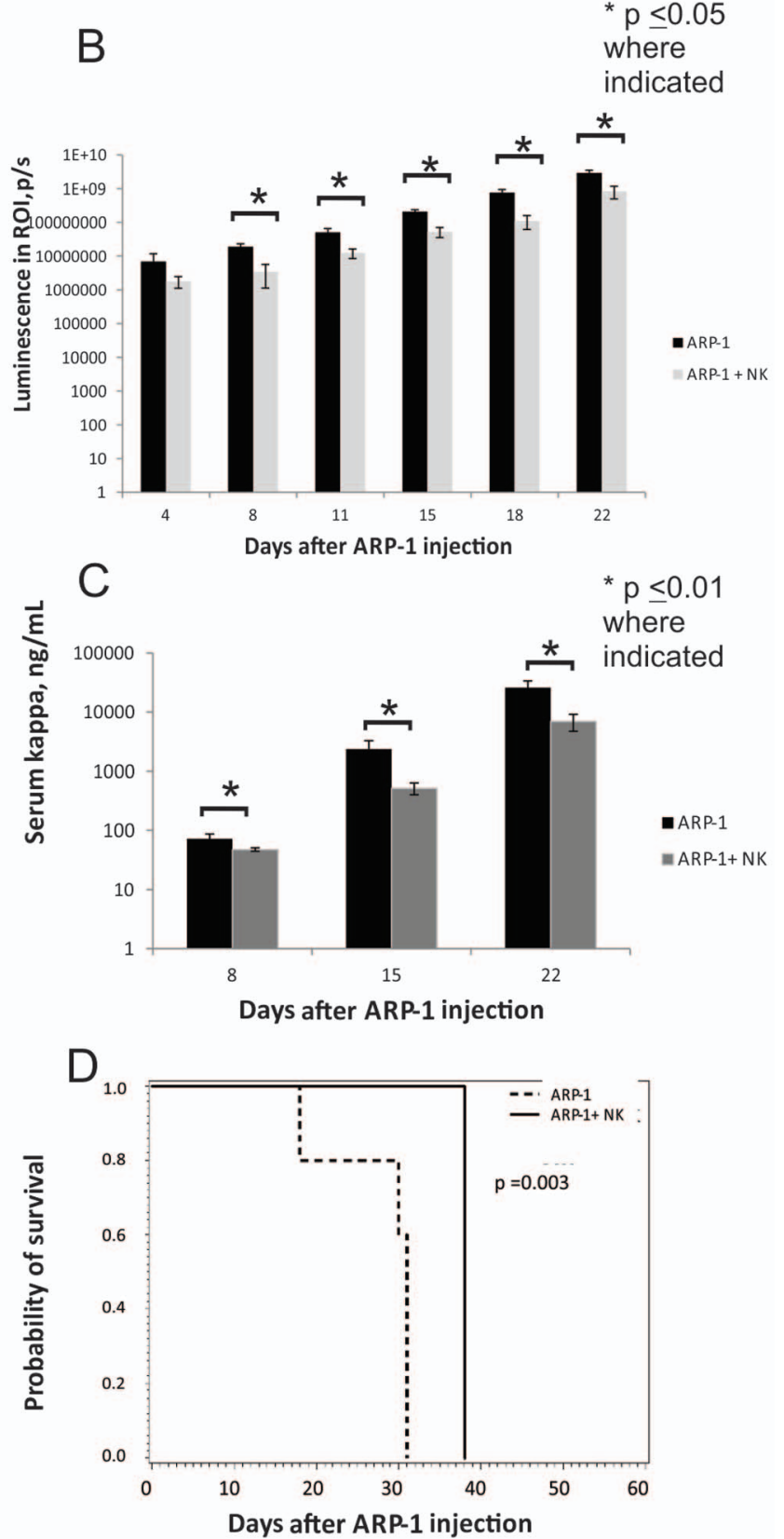

Figure 5. aAPC-expanded CB-NK cells delay development of myeloma in a NSG murine model. $1 \times 10^{6}$ GFP firefly luciferase-transduced ARP-1 cells (Clone 24) were given IV on day -1. In the CB-NK treated group, $10 \times 10^{6}$ ex vivo, aAPC-expanded CB NK cells were given retro-orbitally on days 0,12 and 19 with IL-2, 2000 IU (IP) three times per week. Serial BLI and kappa ELISA measurements were acquired until day 18. Results represent mean values of $n=5$ mice in each group until day 18, by which time 1 mouse in the ARP-1 alone group had died. A. Serial BLI images demonstrate impaired myeloma development in mice receiving CB-NK cells. B. Signal intensity ( $p / s)$ was significantly greater in mice receiving Clone 24 ARP-1 cells alone versus those receiving both Clone 24 ARP-1 cells and CB-NK cells. Region of interest (ROI) is indicated by rectangles superimposed on each mouse from Figure $5 \mathrm{~A}, \mathrm{p} \leq 0.05$ at days $8-22$. C. Serum kappa levels $(\mathrm{ng} / \mathrm{mL})$ were significantly higher in mice treated with Clone 24 ARP-1 cells versus those treated with Clone 24 ARP-1 cells and CB-NK cells, $p \leq 0.01$ at each time point. D. By Kalpan-Meier method, there was a significant difference in survival of the mice, $(p=0.003)$ in favor of the NK-treated group. The mice who received Clone 24 ARP-1 cells alone had a median survival of 31 days versus 38 days for the mice who received Clone 24 ARP-1 cells and CB-NK cells.

doi:10.1371/journal.pone.0076781.g005

Taken together, our results suggest that CB-NK cells are active against MM and can be reliably generated by a GMP-compliant method to obtain clinically relevant doses. Studies are in progress to better determine the role, if any, of KIR-HLA mismatch on NK cell cytotoxicity against primary $\mathrm{CD} 138^{+} \mathrm{MM}$ cells. Finally, a clinical trial using aAPC-expanded CB-NK cells in conjunction with high dose chemotherapy and autologous HSCT for MM is being developed.

\section{Supporting Information}

Figure S1 aAPG-Expanded CB-NK cells displayed equal or more cytotoxicity against $M M$ cells versus $\mathrm{CB}-\mathrm{NK}$ 
cells expanded with IL-2 alone. IL-2 expanded or aAPGexpanded CB-NK cells were co-incubated in triplicate for 4 hours with ${ }^{51} \mathrm{Cr}$-labeled target cells as detailed for Figure 4. Cytotoxicity of aAPC-expanded CB-NK cells was equal to or greater than that of CB-NK cells expanded without aAPCs against various MM cell lines (A: RPMI 8226, B: U266, C: ARP-1; representative data from $n=3$ experiments).

(TIF)

\section{Acknowledgments}

The authors wish to thank Dr. Qing Yi and Jin He for assistance with the multiple myeloma murine model and Wilson Wolf Corporation for

\section{References}

1. Raab MS, Podar K, Breitkreutz I, Richardson PG, Anderson KC (2009) Multiple myeloma. Lancet 374: 324-339.

2. Harousseau JL, Moreau P (2009) Autologous hematopoietic stem-cell transplantation for multiple myeloma. N Engl J Med 360: 2645-2654.

3. Alici E, Konstantinidis KV, Sutlu T, Aints A, Gahrton G, et al. (2007) Antimyeloma activity of endogenous and adoptively transferred activated natural killer cells in experimental multiple myeloma model. Exp Hematol 35: 1839 1846.

4. Garg TK, Szmania SM, Khan JA, Hoering A, Malbrough PA, et al. (2012) Highly activated and expanded natural killer cells for multiple myeloma immunotherapy. Haematologica 97: 1348-1356.

5. Sawanobori M, Suzuki K, Nakagawa Y, Inoue Y, Utsuyama M, et al. (1997) Natural killer cell frequency and serum cytokine levels in monoclonal gammopathies: correlation of bone marrow granular lymphocytes to prognosis. Acta Haematol 98: 150-154.

6. Jurisic V, Srdic T, Konjevic G, Markovic O, Colovic M (2007) Clinical stagedepending decrease of $\mathrm{NK}$ cell activity in multiple myeloma patients. Med Oncol 24: 312-317.

7. PasswegJR, Stern M, Koehl U, Uharek L, Tichelli A (2005) Use of natural killer cells in hematopoetic stem cell transplantation. Bone Marrow Transplant 35: $637-643$.

8. Ruggeri L, Capanni M, Mancusi A, Urbani E, Perruccio K, et al. (2004) Alloreactive natural killer cells in mismatched hematopoietic stem cell transplantation. Blood Cells Mol Dis 33: 216-221.

9. Hsu KC, Gooley T, Malkki M, Pinto-Agnello C, Dupont B, et al. (2006) KIR ligands and prediction of relapse after unrelated donor hematopoietic cell transplantation for hematologic malignancy. Biol Blood Marrow Transplant 12: 828-836.

10. Kroger N, Zabelina T, Berger J, Duske H, Klyuchnikov E, et al. (2011) Donor KIR haplotype B improves progression-free and overall survival after allogeneic hematopoietic stem cell transplantation for multiple myeloma. Leukemia 25: 1657-1661.

11. Willemze R, Ruggeri A, Purtill D, Rodrigues CA, Gluckman E, et al. (2010) Is there an impact of killer cell immunoglobulin-like receptors and KIR-ligand incompatibilities on outcomes after unrelated cord blood stem cell transplantation? Best Pract Res Clin Haematol 23: 283-290.

12. Brunstein CG, Wagner JE, Weisdorf DJ, Cooley S, Noreen H, et al. (2009) Negative effect of KIR alloreactivity in recipients of umbilical cord blood transplant depends on transplantation conditioning intensity. Blood 113: 56285634 .

13. Miller JS, Cooley S, Parham P, Farag SS, Verneris MR, et al. (2007) Missing KIR ligands are associated with less relapse and increased graft-versus-host disease (GVHD) following unrelated donor allogeneic HCT. Blood 109: 50585061 .

14. Kanga U, Mourya M, Seth T, George J, Sood P, et al. (2012) Role of killer immunoglobulin-like receptor-ligand interactions in human leukocyte antigenmatched sibling hematopoietic stem cell transplantation. Transplant Proc 44: 919-921.

15. Fauriat C, Mallet F, Olive D, Costello RT (2006) Impaired activating receptor expression pattern in natural killer cells from patients with multiple myeloma. Leukemia 20: 732-733.

16. Ghiringhelli F, Menard C, Terme M, Flament C, Taieb J, et al. (2005) CD4+CD25+ regulatory $\mathrm{T}$ cells inhibit natural killer cell functions in a transforming growth factor-beta-dependent manner. J Exp Med 202: 10751085 .

17. Tanner J, Tosato G (1991) Impairment of natural killer functions by interleukin 6 increases lymphoblastoid cell tumorigenicity in athymic mice. J Clin Invest 88: 239-247.

18. D'Andrea A, Aste-Amezaga M, Valiante NM, Ma X, Kubin M, et al. (1993) Interleukin 10 (IL-10) inhibits human lymphocyte interferon gamma-production by suppressing natural killer cell stimulatory factor/IL-12 synthesis in accessory cells. J Exp Med 178: 1041-1048. providing GP500 bioreactors. We would also like to thank the MD Anderson Cord Blood Bank and Myeloma Tissue Bank for the normal and malignant cells used in these studies.

\section{Author Contributions}

Conceived and designed the experiments: NS BMA HY SK DL LC WD SL TS SP JG KR EY AN JB IK CB EJS. Performed the experiments: NS BMA HY SK WD SL TS EY AN JB IK. Analyzed the data: NS BMA HY SK WD SL SR TS SP JG MW KR EY AN JB IK CB EJS. Contributed reagents/materials/analysis tools: DL LC JG MW AN JB RC CB EJS. Wrote the paper: NS BMA HY LC WD KR EY AN IK RC CB EJS.

19. Tinhofer I, Marschitz I, Henn T, Egle A, Greil R (2000) Expression of functional interleukin-15 receptor and autocrine production of interleukin-15 as mechanisms of tumor propagation in multiple myeloma. Blood 95: 610-618.

20. Godfrey J, Benson DM Jr. (2012) The role of natural killer cells in immunity against multiple myeloma. Leuk Lymphoma 53: 1666-1676.

21. Alici E, Sutlu T, Bjorkstrand B, Gilljam M, Stellan B, et al. (2008) Autologous antitumor activity by NK cells expanded from myeloma patients using GMPcompliant components. Blood 111: 3155-3162.

22. Katodritou E, Terpos E, North J, Kottaridis P, Verrou E, et al. (2011) Tumorprimed natural killer cells from patients with multiple myeloma lyse autologous, NK-resistant, bone marrow-derived malignant plasma cells. Am J Hematol 86: 967-973.

23. Carbone E, Neri P, Mesuraca M, Fulciniti MT, Otsuki T, et al. (2005) HLA class I, NKG2D, and natural cytotoxicity receptors regulate multiple myeloma cell recognition by natural killer cells. Blood 105: 251-258.

24. Xing D RA, Gribben JG, Decker WK, Burks JK, Li S, et al. (2010) Cord Blood Natural Killer Cells Exhibit Defective Lytic Immunological Synapse Formation that is Reversed with IL-2 Ex Vivo Expansion. J Immunother 33(7): 684-96.

25. Lapteva N, Durett AG, Sun J, Rollins LA, Huye LL, et al. (2012) Large-scale ex vivo expansion and characterization of natural killer cells for clinical applications. Cytotherapy 14: 1131-1143.

26. Denman CJ, Senyukov VV, Somanchi SS, Phatarpekar PV, Kopp LM, et al. (2012) Membrane-bound IL-21 promotes sustained ex vivo proliferation of human natural killer cells. PLoS One 7: e30264.

27. Hoyos V, Savoldo B, Quintarelli C, Mahendravada A, Zhang M, et al. (2010) Engineering CD19-specific T lymphocytes with interleukin-15 and a suicide gene to enhance their anti-lymphoma/leukemia effects and safety. Leukemia 24: $1160-1170$.

28. Savoldo B, Rooney CM, Di Stasi A, Abken H, Hombach A, et al. (2007) Epstein Barr virus specific cytotoxic $\mathrm{T}$ lymphocytes expressing the anti-CD30zeta artificial chimeric T-cell receptor for immunotherapy of Hodgkin disease. Blood 110: 2620-2630.

29. Yang J, Cao Y, Hong S, Li H, Qian J, et al. (2009) Human-like mouse models for testing the efficacy and safety of anti-beta2-microglobulin monoclonal antibodies to treat myeloma. Clin Cancer Res 15: 951-959.

30. Feinman R, Koury J, Thames M, Barlogie B, Epstein J, et al. (1999) Role of NFkappaB in the rescue of multiple myeloma cells from glucocorticoid-induced apoptosis by bcl-2. Blood 93: 3044-3052.

31. Ramsay AG, Johnson AJ, Lee AM, Gorgun G, Le Dieu R, et al. (2008) Chronic lymphocytic leukemia $\mathrm{T}$ cells show impaired immunological synapse formation that can be reversed with an immunomodulating drug. J Clin Invest 118: 24272437.

32. Gill S, Vasey AE, De Souza A, Baker J, Smith AT, et al. (2012) Rapid development of exhaustion and down-regulation of eomesodermin limit the antitumor activity of adoptively transferred murine natural killer cells. Blood 119: $5758-5768$.

33. Intlekofer AM, Takemoto N, Wherry EJ, Longworth SA, Northrup JT, et al. (2005) Effector and memory CD8+ $\mathrm{T}$ cell fate coupled by T-bet and eomesodermin. Nat Immunol 6: 1236-1244.

34. Stinchcombe JC, Bossi G, Booth S, Griffiths GM (2001) The immunological synapse of CTL contains a secretory domain and membrane bridges. Immunity 15: $751-761$.

35. Orange JS (2008) Formation and function of the lytic NK-cell immunological synapse. Nat Rev Immunol 8: 713-725.

36. Bjorkstrand B, Iacobelli S, Hegenbart U, Gruber A, Greinix H, et al. (2011) Tandem autologous/reduced-intensity conditioning allogeneic stem-cell transplantation versus autologous transplantation in myeloma: long-term follow-up. J Clin Oncol 29: 3016-3022.

37. Krishnan A, Pasquini MC, Logan B, Stadtmauer EA, Vesole DH, et al. (2011) Autologous haemopoietic stem-cell transplantation followed by allogeneic or autologous haemopoietic stem-cell transplantation in patients with multiple myeloma (BMT CTN 0102): a phase 3 biological assignment trial. Lancet Oncol 12: 1195-1203. 
38. Rosinol L, Perez-Simon JA, Sureda A, de la Rubia J, de Arriba F, et al. (2008) A prospective PETHEMA study of tandem autologous transplantation versus autograft followed by reduced-intensity conditioning allogeneic transplantation in newly diagnosed multiple myeloma. Blood 112: 3591-3593.

39. Giaccone L, Storer B, Patriarca F, Rotta M, Sorasio R, et al. (2011) Long-term follow-up of a comparison of nonmyeloablative allografting with autografting for newly diagnosed myeloma. Blood 117: 6721-6727.

40. Ruggeri L, Capanni M, Urbani E, Perruccio K, Shlomchik WD, et al. (2002) Effectiveness of donor natural killer cell alloreactivity in mismatched hematopoietic transplants. Science 295: 2097-2100.

41. Lundqvist A, McCoy JP, Samsel L, Childs R (2007) Reduction of GVHD and enhanced antitumor effects after adoptive infusion of alloreactive Ly49mismatched NK cells from MHC-matched donors. Blood 109: 3603-3606.

42. Shi J, Tricot G, Szmania S, Rosen N, Garg TK, et al. (2008) Infusion of haploidentical killer immunoglobulin-like receptor ligand mismatched NK cells for relapsed myeloma in the setting of autologous stem cell transplantation. Br J Haematol 143: 641-653.

43. Roda-Navarro P, Vales-Gomez M, Chisholm SE, Reyburn HT (2006) Transfer of NKG2D and MICB at the cytotoxic NK cell immune synapse correlates with a reduction in NK cell cytotoxic function. Proc Natl Acad Sci U S A 103: 11258-11263.

44. Paley MA, Gordon SM, Bikoff EK, Robertson EJ, Wherry EJ, et al. (2012) Technical Advance: Fluorescent reporter reveals insights into eomesodermin biology in cytotoxic lymphocytes. J Leukoc Biol.
45. Townsend MJ, Weinmann AS, Matsuda JL, Salomon R, Farnham PJ, et al. 2004) T-bet regulates the terminal maturation and homeostasis of NK and Valpha14i NKT cells. Immunity 20: 477-494.

46. Spanholtz J, Tordoir M, Eissens D, Preijers F, van der Meer A, et al. (2010) High log-scale expansion of functional human natural killer cells from umbilical cord blood CD34-positive cells for adoptive cancer immunotherapy. PLoS One 5: e9221.

47. Spanholtz J, Preijers F, Tordoir M, Trilsbeek C, Paardekooper J, et al. (2011) Clinical-grade generation of active NK cells from cord blood hematopoietic progenitor cells for immunotherapy using a closed-system culture process. PLoS One 6: e20740.

48. Beck RC, Padival M, Yeh D, Ralston J, Cooke KR, et al. (2009) The Notch ligands Jagged2, Delta1, and Delta4 induce differentiation and expansion of functional human NK cells from CD34+ cord blood hematopoietic progenitor cells. Biol Blood Marrow Transplant 15: 1026-1037.

49. Rocha V, Labopin M, Sanz G, Arcese W, Schwerdtfeger R, et al. (2004) Transplants of umbilical-cord blood or bone marrow from unrelated donors in adults with acute leukemia. N Engl J Med 351: 2276-2285.

50. Laughlin MJ, Eapen M, Rubinstein P, Wagner JE, Zhang MJ, et al. (2004) Outcomes after transplantation of cord blood or bone marrow from unrelated donors in adults with leukemia. N Engl J Med 351: 2265-2275.

51. Anasetti C, Aversa F, Brunstein CG (2012) Back to the future: mismatched unrelated donor, haploidentical related donor, or unrelated umbilical cord blood transplantation? Biol Blood Marrow Transplant 18: S161-165.

52. Gluckman E, Ruggeri A, Volt F, Cunha R, Boudjedir K, et al. (2011) Milestones in umbilical cord blood transplantation. Br J Haematol 154: 441-447. 